Méndez-Giménez, A.; García-Romero, C. y Cecchini-Estrada, J. A. (2018). Metas de logro 3×2, amistad y afecto en educación física: diferencias edad-sexo / 3×2 Achievement Goals, Friendship and Affectivity in Physical Education: Age-Gender Differences. Revista Internacional de Medicina y Ciencias de la Actividad Física y el Deporte vol. 18 (72) pp. 637-653. Http://cdeporte.rediris.es/revista/revista72/artmoverse975.htm

DOI: http://doi.org/10.15366/rimcafd2018.72.003

\title{
ORIGINAL
}

\section{METAS DE LOGRO 3×2, AMISTAD Y AFECTO EN EDUCACIÓN FÍSICA: DIFERENCIAS EDAD-SEXO}

\section{$3 \times 2$ ACHIEVEMENT GOALS, FRIENDSHIP AND AFFECTIVITY IN PHYSICAL EDUCATION: AGE-GENDER DIFFERENCES}

\author{
Méndez-Giménez, A. ${ }^{1}$; García-Romero, C. ${ }^{2}$ y Cecchini-Estrada, J. A. ${ }^{3}$ \\ 1 Profesor Titular de Universidad. Facultad de Formación del Profesorado y Educación. \\ Universidad de Oviedo (España) mendezantonio@uniovi.es \\ 2 Doctoranda de la Universidad de Oviedo (España) UO211054@uniovi.es \\ ${ }^{3}$ Catedrático de Universidad. Facultad de Formación del Profesorado y Educación. Universidad \\ de Oviedo (España) cecchini@uniovi.es
}

Código UNESCO / UNESCO code: 5899. EF y Deporte / PE and Sport Clasificación del Consejo de Europa / Council of Europe classification: 4. Educación Física y Deporte comparado / Compared Sport and Physical Education; 5. Didáctica y metodología / Didactic and methodology.15. Psicología del deporte / Sport Psychology

Recibido 19 de diciembre de 2016 Received December 19, 2016 Aceptado 13 de septiembre de 2017 Accepted September 13, 2017

\section{RESUMEN}

Se persiguen tres objetivos: (a) analizar las diferencias relativas a la edad y sexo en las metas de logro 3x2, metas de amistad, y afectividad en Educación Física, (b) estudiar las relaciones entre esas variables, y (c) explorar el valor predictivo de las metas de logro $3 \times 2$ y amistad sobre la afectividad. La muestra estuvo formada por 1610 alumnos (855 varones y 755 mujeres) de 10 a 17 años. Se realizaron MANOVAS, análisis univariados (Scheffé), y regresiones lineales. Los resultados mostraron un descenso significativo de las metas de logro (excepto aproximación-otro), metas de amistad y afecto positivo debido al desarrollo. Los varones puntuaron más alto las metas de aproximación-tarea, aproximación-otro, y evitación-otro. Las metas de aproximación-tarea y aproximación-amistad fueron los principales predictores positivos del afecto positivo. La franja de 13 a 14 años muestra una gran sensibilidad a los tres estándares de competencia de logro, competencia social e inestabilidad afectiva. 
PALABRAS CLAVE: Metas de logro; edad; sexo; Educación Física.

\section{ABSTRACT}

The objectives of the study are three: (a) to analyze the differences related to age and sex in the $3 \times 2$ achievement goals, the friendship goals, and the affectivity in Physical Education, (b) to study the relationships between these variables, and c) to explore the predictive value of the $3 \times 2$ achievement goals and friendship on affectivity. The sample consisted of 1610 students ( 855 males and 755 females), aged between 10 and 17 years. MANOVAS, univariate analyzes (Scheffé), and linear regressions were performed. The results showed a significant decrease in achievement goals (except other-approach), friendship goals, and positive affect due to development. Males scored higher on taskapproach, other-approach, and other-avoidance goals than women. Taskapproach and friendship-approach were the main positive predictors of positive affect. The range of 13 to 14 years shows a great sensitivity to the three competences of achievement, social competence, and affective instability.

KEY WORD: Achievement goal; age; gender; Physical Education.

\section{INTRODUCCIÓN}

El constructo de metas logro se desarrolló a finales de la década de los 70 por autores como Ames (1984), Dweck (1986), Maehr (1989) y Nicholls (1984). Todos ellos, concibieron las metas de logro como las razones o propósitos que dirigen el comportamiento de las personas (Ames, 1992). Esta teoría ha evolucionado desde entonces hasta la actualidad, pasando por cuatro modelos diferentes: dicotómico, tricotómico, modelo $2 \times 2$ y, el más reciente, el modelo $3 \times 2$. En un principio, las orientaciones de meta se conceptualizaron de forma dicotómica; por un lado, las metas de maestría, cuyo objetivo era desarrollar la competencia hacia la tarea y, por otro, las metas de rendimiento, cuyo fin consistía en demostrar competencia en general (Ames, 1992). En años sucesivos, se derivó al modelo tricotómico, al incluir, además de la definición (maestría y rendimiento), la valencia de la competencia en las metas de rendimiento (aproximación y evitación). El foco de la aproximación era el éxito y los estados positivos, mientras que el de la evitación era el fracaso e intentar alejarse de la posibilidad negativa (Elliot \& Harackiewicz, 1996). En consecuencia, el modelo conceptualizó tres tipos de metas: maestría, aproximación-rendimiento y evitación-rendimiento (Elliot \& Church, 1997).

En una evolución posterior, Elliot \& McGregor (2001) especularon sobre las metas de evitación-maestría. El cruce de la definición y los tipos de valencia de la competencia dio lugar a las cuatro metas de logro que componen el modelo 2x2: aproximación-maestría, evitación-maestría, aproximación-rendimiento y evitación-rendimiento. Las metas de aproximación-maestría se correspondían con la concepción tradicional de la meta de maestría, mientras que las metas de evitación-maestría pretenden evitar la falta de aprendizaje. Por su parte, las 
metas de aproximación-rendimiento se relacionaron con las metas de rendimiento tradicional, y las metas de evitación-rendimiento se refirieron a evitar hacerlo peor que los demás.

La última evolución ha conformado el marco de metas de logro 3x2 (Elliot, Murayama \& Pekrun, 2011). Este reciente impulso propone bifurcar las metas de maestría al considerar dos estándares diferenciados: tarea y yo. En las metas basadas en la tarea, la competencia se conceptualiza en términos de hacerlo bien o mal en relación a una tarea. Sin embargo, en las metas basadas en el yo, es el propio foco personal el referente de evaluación. En consecuencia, las personas que adoptan estas metas se centran en hacerlo bien o mal en relación a como lo han hecho en el pasado o como lo pueden hacer en el futuro. Al cruzar los tres estándares (definición) con las dos formas en que la competencia puede ser valenciada (valencia), se proponen seis metas de logro: metas de aproximación-tarea (centradas en el logro de la competencia basada en la tarea), metas de evitación-tarea (centradas en la evitación de la incompetencia basada en la tarea), metas de aproximación-yo (centradas en el logro de competencia basada en el yo), metas evitación-yo (centradas en la evitación de incompetencia basada en yo), metas aproximación-otro (centradas en el logro de competencia basada en el otro) y metas evitación-otro (centradas en la evitación de incompetencia basada en el otro).

Estos nuevos avances han suscitado un enorme interés entre los investigadores, principalmente en la búsqueda de patrones de relación entre las metas de logro $3 \times 2$ y variables de resultado motivacionales. En el contexto de la educación general, Elliot et al. (2011) encontraron consistentes relaciones adaptativas de las metas de aproximación-tarea (e.g., con motivación intrínseca, eficacia del aprendizaje), y más moderadas de las metas de aproximación-yo (e.g., con energía en clase) y aproximación-otro (e.g., rendimiento en examen, eficacia del aprendizaje). Las metas de evitación-yo fueron desadaptativas (relación negativa con absorción y energía en clase), al igual que las metas de evitación-otro (relaciones negativas con rendimiento en exámenes y motivación intrínseca, y positiva con preocupación en los exámenes). En otro estudio, Brondino, Raccanello, \& Pasini (2014) reportaron que las metas de aproximación-tarea predijeron positivamente las emociones positivas y, negativamente, las negativas. Las metas de aproximación-yo solo predijeron positivamente la diversión. Las metas de evitación-tarea predijeron negativamente las emociones positivas, y las de evitación-otro predijeron positivamente las emociones positivas (diversión, esperanza, orgullo). Las metas de aproximación-otro y evitación-yo no predijeron ninguna emoción. Más tarde, Diseth (2015) concluyó que las metas de aproximación-tarea, pero también las metas de aproximación-otro, se relacionaron con los aspectos más funcionales de las variables motivacionales (e.g., autoeficacia, estrategias de aprendizaje), mientras que las metas basadas en el yo (tanto de aproximación como de evitación) mostraron un patrón de relación inverso (e.g., menos logro académico o estrategias de aprendizaje). Por tanto, en comparación con la literatura previa, el estudio de Diseth (2015) cuestionó las presumidas relaciones positivas de las metas de aproximación-yo y puso en valor supuestos efectos positivos de las metas de aproximación-otro. Recientemente, Méndez-Giménez, Cecchini, 
Fernández-Río, Méndez-Alonso, \& Prieto-Saborit (2017) confirmaron parcialmente el patrón de resultados de Elliot et al (2011). Las metas de aproximación-tarea y aproximación-yo se relacionaron positivamente con resultados positivos (autodeterminación y satisfacción con la vida). Las metas de aproximación-otro se asociaron positivamente con variables de valencia positiva (e.g., satisfacción con la vida), pero también negativamente con variables positivas (e.g., índice de autodeterminación). Las metas de evitación-otro se vincularon negativamente con resultados positivos (e.g., satisfacción con la vida). Finalmente, las de metas de evitación-tarea presentaron un patrón de relaciones ligeramente negativo (índice de autodeterminación) y nulo (satisfacción con la vida).

En el contexto de la EF, Méndez-Giménez, Cecchini, \& Fernández-Río (2014) replicaron ese patrón más adaptativo de las metas de aproximación-tarea (relación positiva con índice de autodeterminación, competencia percibida, dimensiones de habilidad y condición física del auto-concepto físico, y metas de aproximación-amistad). Las metas de aproximación-otro ocuparon un segundo lugar en el nivel adaptativo de las variables a estudio (relaciones positivas con el índice de autodeterminación, competencia percibida, y las dimensiones de habilidad, atractivo físico, y condición física del auto-concepto físico). Sin embargo, las metas de aproximación-yo solo tuvieron relación positiva con el índice de autodeterminación. En el ámbito deportivo, Mascret, Elliot, \& Cury (2015) mostraron relaciones positivas de las metas de aproximación-tarea con un número superior de variables de consecuencia (e.g., interés intrínseco y competencia percibida) que las metas de aproximación-yo (solo con interés intrínseco), y que las metas de aproximación-otro (solo con competencia percibida).

Pese a la evolución teórica del marco en los diferentes ámbitos de estudio, las variables edad y sexo de los participantes aún no han sido investigadas desde la perspectiva de metas de logro 3×2. En relación al desarrollo de las metas de logro, Paulick, Watermann, \& Nückles (2013) consideraron dos posibles vías de teoría e investigación. Por un lado, los psicólogos del desarrollo (Dweck, 2000; Nicholls, 1984) argumentaron que las metas de maestría de los estudiantes disminuyen, y las metas de rendimiento aumentan en la adolescencia, principalmente como consecuencia del desarrollo cognitivo y su concepto de habilidad que se vuelve cada vez más diferenciado. Por otro, los psicólogos educativos sugieren que la transición de las escuelas primarias a los centros de secundaria son la principal fuente de disminución general de la motivación durante la adolescencia temprana (Juvonen, Le, Kaganoff, Augustine, \& Constant, 2004). Así, diversos estudios (e.g., Bong, 2009; Shim, Ryan \& Anderson, 2008) confirmaron un descenso general en todos los tipos de metas de logro $2 \times 2$. Es preciso esclarecer cómo afecta el desarrollo personal en la adopción de las metas de logro desde la perspectiva del nuevo modelo 3x2.

Finalmente, numerosos trabajos han enfatizado la necesidad de incluir las metas sociales en los estudios motivacionales (Garn \& Sun, 2009; Cecchini et al., 2008; Cecchini, González, Méndez-Giménez \& Fernández-Río, 2011; Méndez-Giménez, Fernández-Río \& Cecchini, 2012; 2014; 2015). Méndez- 
Giménez et al. (2015) examinaron el valor predictivo de las metas de logro 2x2 y las metas de amistad sobre diversas consecuencias motivacionales en el contexto de la EF, y encontraron que las metas de aproximación-maestría y evitación-amistad ejercieron como los únicos predictores positivos del esfuerzo, mientras que las metas de aproximación-maestría y aproximación-amistad se revelaron como los únicos predictores positivos de la diversión. En la actualidad, existe una laguna de investigación en relación a esta temática desde marco teórico de metas de logro $3 \times 2$.

Considerando estos antecedentes, el presente estudio se propuso tres objetivos: (a) analizar las diferencias relativas a la edad y el sexo en las metas de logro 3x2, las metas de amistad (aproximación y evitación), y la afectividad en el contexto de la Educación Física, (b) estudiar las relaciones entre las variables a estudio, y (c) explorar el valor predictivo conjunto de metas de logro $3 \times 2$ y las metas de amistad en la afectividad en función de la edad.

\section{MÉTODO}

\section{PARTICIPANTES}

Se realizó un muestreo intencional o de conveniencia. La muestra estaba formada por 1610 estudiantes, 855 varones y 755 mujeres, pertenecientes a 60 aulas de 7 colegios e institutos del territorio nacional, con edades comprendidas entre 10 y 17 años de edad. De ellos, 225 eran de $5^{\circ}$ y $6^{\circ}$ de Educación Primaria, 341 de $1^{\circ}$ de ESO, 411 de $2^{\circ}$ de ESO, 272 de $3^{\circ}$ de ESO, 240 de $4^{\circ}$ de ESO, y 121 de $1^{\circ}$ de Bachillerato. La Tabla 1 presenta la distribución considerando edad y sexo de los participantes. Para examinar las tendencias en el desarrollo, se fusionaron los datos de dos grupos adyacentes formando así tres tramos de edad.

Tabla 1. Distribución de la muestra por tramos de edad y sexo

\begin{tabular}{llll}
\hline & \multicolumn{2}{l}{ Sexo } & \multicolumn{1}{l}{ Total } \\
\cline { 2 - 4 } 10-12 años & Hombre & Mujer & \\
$13-14$ años & $365(56.1 \%)$ & $210(51.9 \%)$ & 405 \\
$15-17$ años & $295(52.8 \%)$ & $281(43.5 \%)$ & 646 \\
& $264(47.2 \%)$ & 559 \\
\hline
\end{tabular}

\section{INSTRUMENTOS}

Metas de logro 3x2. Se utilizó el cuestionario de Metas de Logro en Educación Física (CML 3×2-EF) de Méndez-Giménez, Cecchini et al. (2014). Se trata de la validación al castellano y al contexto específico de la EF del cuestionario desarrollado por Elliot et al. (2011). Los ítems fueron precedidos por el encabezamiento "En las clases de Educación Física mi meta es...". Este instrumento está compuesto por un total de 24 ítems agrupados en 6 factores: aproximación-tarea (e.g. "...realizar correctamente muchos ejercicios y habilidades"), evitación-tarea (e.g. "...evitar hacer mal las tareas"), aproximaciónyo (e.g. "...realizar los ejercicios mejor de lo que lo hago habitualmente"), evitación-yo (e.g. "...evitar hacer las habilidades peor de como lo hago habitualmente"), aproximación-otro (e.g. "...superar a otros estudiantes en la 
realización de habilidades y tareas"), evitación-otro (e.g. "...evitar hacer peor los ejercicios y las tareas que los otros estudiantes"). Los alfa de Cronbach del estudio de Méndez-Giménez, Cecchini et al. (2014), oscilaron entre .74 (evitación-tarea) y .89 (aproximación-otro). Los participantes indicaron el grado de acuerdo con cada una de esas afirmaciones mediante una escala Likert de 5 puntos, que va desde 1 (nada cierto para mí) a 5 (totalmente cierto para mí).

Metas de Amistad. Se empleó la versión validada al castellano por MéndezGiménez, Fernández-Río et al. (2014) del Cuestionario Relationship Goals Questionnaire-Friendship Version (RGQ-F) de Elliot, Gables y Mapes (2006). Los ítems vienen precedidos por la siguiente introducción: "En mis clases de EF trato de...". La escala está compuesta por 8 ítems agrupados en dos dimensiones: aproximación-amistad (4 ítems), (e.g. "...profundizar en las relaciones con mis amigos") y evitación-amistad (4 ítems), (e.g. "...evitar desacuerdos y conflictos con mis amigos"). El coeficiente de fiabilidad compuesta de este instrumento en el estudio de Méndez-Giménez, Fernández-Río et al. (2014) fue de .86 y .85 para las metas de aproximación-amistad y evitación-amistad, respectivamente. Los participantes indicaron el grado de acuerdo con cada una de esas afirmaciones mediante una escala Likert de 5 puntos, que va desde 1 (totalmente en desacuerdo) a 5 (totalmente de acuerdo).

Afectividad. Se utilizó el cuestionario PANASN (Afecto Positivo y Negativo para Niños y Adolescentes), un instrumento adaptado a la población escolar por Sandín (2003) a partir de la versión para adultos del PANAS (Positive and Negative Affect Schedule). Los ítems fueron precedidos por el encabezamiento: "En mis clases de Educación Física...". EI PANASN constituye un cuestionario de autoinforme formado por 20 ítems, diez ítems evalúan el afecto positivo (e. g. "...soy una persona animada, suelo emocionarme") y los otros diez, el afecto negativo (e. g. "...me siento nervioso"). En el estudio de Sandín (2003), los coeficientes alfa de Cronbach para los varones fueron de .73 y .74 para el afecto positivo y negativo, respectivamente, mientras que los valores para las mujeres fueron de .72 y .75, respectivamente. Los participantes indicaron el grado de acuerdo con cada una de esas afirmaciones mediante una escala Likert de 3 puntos, que va desde 1 (nunca) a 3 (muchas veces).

\section{PROCEDIMIENTO}

En un primer momento, se contactó con los directores de los centros escolares para solicitar su colaboración en este estudio y pedir el consentimiento informado de los padres de los estudiantes. Antes de distribuir los cuestionarios al alumnado, se habló con los maestros y profesores de Educación Física para explicarles el protocolo que debían seguir y rellenarlos, bien en formato papel, bien online, a través de la plataforma Google Forms. Se decidió que en 6o de Educación Primaria, dada la complejidad del instrumento, los maestros y maestras leyeran uno a uno los ítems, mientras que en Secundaria y Bachillerato los cubrieron de forma independiente. Los cuestionarios se completaron en el aula ordinaria o de informática, según el método de administración elegido, durante los meses de abril y mayo de 2015. La participación del alumnado fue 
voluntaria y se insistió en la confidencialidad de las respuestas, ya que estas, no afectarían a la nota de la asignatura.

\section{ANÁLISIS DE DATOS}

En primer lugar, se realizaron análisis descriptivos (media y desviación típica), análisis de consistencia interna, mediante alfa de Cronbach y correlaciones bivariadas para cada tramo de edad. Para observar la evolución de las metas de logro 3x2 en función de la edad y del sexo se realizó un análisis multivariante de la varianza (MANOVA), con estas variables como factores intrasujeto. Cuando se detectaron diferencias significativas en el MANOVA se llevaron a cabo análisis univariados empleando el test de Scheffé. Dentro de cada grupo de edad, se realizaron pruebas $t$ para muestras relacionadas en todos los pares posibles de las metas de logro $3 \times 2$. Por último, se ejecutaron varias regresiones lineales para examinar las metas de logro $3 \times 2$ y las metas de amistas como predictores de las variables dependientes (afectividad positiva y negativa).

\section{RESULTADOS}

\section{Análisis descriptivos, fiabilidad de las escalas y correlaciones bivariadas}

La Tabla 2 presenta las medias, desviaciones típicas, y alfas de Cronbach de todas las variables de interés en función de los grupos de edad. Los niveles de fiabilidad fueron aceptables en todas las variables y franjas de edad (Nunnally, 1978). Se observó una primera tendencia relativa al desarrollo: los coeficientes $\alpha$ de Cronbach del grupo más joven fueron más bajos que los de los otros dos grupos de mayor edad.

Tabla 2. Análisis descriptivos y fiabilidad de los datos de la muestra total y por grupos de edad

\begin{tabular}{|c|c|c|c|c|c|c|c|c|c|c|c|c|}
\hline & \multicolumn{3}{|c|}{$\begin{array}{l}\text { Muestra total } \\
(n=1610)\end{array}$} & \multicolumn{3}{|c|}{$\begin{array}{c}\text { 10-12 años } \\
(\mathrm{n}=405)\end{array}$} & \multicolumn{3}{|c|}{$\begin{array}{c}\text { 13-14 años } \\
(\mathrm{n}=646)\end{array}$} & \multicolumn{3}{|c|}{$\begin{array}{c}\text { 15-17 años } \\
(\mathrm{n}=559)\end{array}$} \\
\hline & $M$ & $D T$ & $\alpha$ & $M$ & $D T$ & $\alpha$ & $M$ & $D T$ & 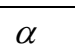 & $M$ & $D T$ & \\
\hline $1 . f$ & 4.13 & .78 & 8 & $4.29^{a}$ & .71 & 7 & 4.13 & .78 & & $4.01^{\mathrm{c}}$ & .80 & 33 \\
\hline & & .94 & .7 & & .94 & & & .94 & & & .9 & .7 \\
\hline & & .80 & & & .70 & & & .8 & & & .8 & 3 \\
\hline & 3 & 1.0 & .7 & 3.8 & 1.0 & & & 1.0 & & & .9 & .82 \\
\hline & & & .88 & 3. & 1. & .8 & & 1.14 & .8 & $3.22^{\mathrm{a}}$ & 1.1 & .91 \\
\hline & 3.43 & 1.11 & .83 & $3.56^{a}$ & 1.10 & $.7 \mathrm{~s}$ & & 1.11 & .8 & $3.33^{b}$ & 1.09 & .85 \\
\hline & & .93 & .8 & & .87 & & & .92 & 0 & & .9 & 8 \\
\hline & 3.97 & .92 & .75 & $4.22^{\mathrm{a}}$ & .86 & .7 & 3.9 & .93 & . & $3.82^{b}$ & .9 & .76 \\
\hline 9. $\mathrm{A}$ & 2.41 & .35 & .74 & $2.45^{\mathrm{a}}$ & .33 & .71 & $2.38^{b}$ & .37 & .77 & $2.39^{b}$ & .33 & .74 \\
\hline 10 & 1.76 & .43 & .81 & $1.74^{\mathrm{a}}$ & .43 & .80 & $1.77^{a}$ & .43 & .81 & $1.75^{a}$ & .42 & .81 \\
\hline
\end{tabular}

Emergió un segundo patrón también asociado a la edad en todas las variables: los valores más altos se dieron entre los estudiantes más jóvenes. Destacó la elevada puntuación de las metas de aproximación-tarea en los tres grupos $(4.29,4.13$ y 4.01 , respectivamente) y aproximación-yo $(4.28,4.03$ y 3.99 , 
respectivamente), y la más baja valoración de las metas de aproximación-otro. En cuanto a las metas de amistad, la dimensión evitación obtuvo valores ligeramente superiores a la aproximación en todos los grupos. Por último, el afecto positivo obtuvo valores notablemente más elevados que el afecto negativo.

Las Tablas 3 y 4 presentan los coeficientes de correlación bivariadas dentro de cada grupo de edad. Varios aspectos son destacables. En primer lugar, los coeficientes de correlación entre las seis metas de logro fueron generalmente más pequeños en la muestra más joven, con respecto a las otras dos franjas de edad. Además, en los tres grupos las correlaciones más elevadas se localizan entre las metas de aproximación-tarea y aproximación-yo $(.69, .71$ y, .75), y entre las metas de evitación-tarea y evitación-yo $(.68, .73$ y, .68). En relación a las metas de amistad, sus coeficientes de correlación aumentaron con la edad (.61, $.63 \mathrm{y}, .64$, respectivamente). Finalmente, no existieron correlaciones entre las dos dimensiones de afectividad.

Tabla 3. Correlaciones bivariadas para los grupos 1 (10-12 años) y 2 (13-14 años)

\begin{tabular}{llllllllllll}
\hline & 1 & 2 & 3 & 4 & 5 & 6 & 7 & 8 & 9 & 10 & 11 \\
\hline 1. Sexo & 1 & $-.12^{* *}$ & -.05 & -.06 & $-.08^{*}$ & $-.19^{* *}$ & $-.13^{* *}$ & .04 & .02 & $-.09^{*}$ & .03 \\
2. Ap-Tarea & -.04 & 1 & $.58^{* *}$ & $.71^{* *}$ & $.56^{* *}$ & $.32^{* *}$ & $.34^{* *}$ & $.38^{* *}$ & $.38^{* *}$ & $.40^{* *}$ & $-.10^{* *}$ \\
3. Ev-Tarea & .09 & $.45^{* *}$ & 1 & $.53^{* *}$ & $.73^{* *}$ & $.17^{* *}$ & $.47^{* *}$ & $.24^{* *}$ & $.37^{* *}$ & $.31^{* *}$ & $-.10^{* *}$ \\
4. Ap-Yo & .00 & $.69^{* *}$ & $.44^{* *}$ & 1 & $.65^{* *}$ & $.33^{* *}$ & $.37^{* *}$ & $.37^{* *}$ & $.38^{* *}$ & $.37^{* *}$ & $-.08^{*}$ \\
5. Ev-Yo & .06 & $.42^{* *}$ & $.68^{* *}$ & $.48^{* *}$ & 1 & $.31^{* *}$ & $.56^{* *}$ & $.20^{* *}$ & $.34^{* *}$ & $.27^{* *}$ & -.03 \\
6. Ap-Otro & $-.20^{* *}$ & $.27^{* *}$ & $.10^{*}$ & $.22^{* *}$ & $.21^{* *}$ & 1 & $.66^{* *}$ & $.20^{* *}$ & $.26^{* *}$ & $.21^{* *}$ & $.12^{* *}$ \\
7. Ev-Otro & $-.10^{*}$ & $.32^{* *}$ & $.46^{* *}$ & $.27^{* *}$ & $.51^{* *}$ & $.60^{* *}$ & 1 & $.20^{* *}$ & $.36^{* *}$ & $.18^{* *}$ & .06 \\
8. Ap-Amistad & -.05 & $.40^{* *}$ & $.20^{* *}$ & $.36^{* *}$ & $.15^{* *}$ & $.17^{* *}$ & $.21^{* *}$ & 1 & $.63^{* *}$ & $.26^{* *}$ & $.10^{*}$ \\
9. Ev-Amistad & .06 & $.44^{* *}$ & $.45^{* *}$ & $.43^{* *}$ & $.34^{* *}$ & $.10^{*}$ & $.30^{* *}$ & $.61^{* *}$ & 1 & $.27^{* *}$ & .04 \\
10. A. Positivo & -.05 & $.38^{* *}$ & $.30^{* *}$ & $.32^{* *}$ & $.26^{* *}$ & $.17^{* *}$ & $.26^{* *}$ & $.32^{* *}$ & $.30^{* *}$ & 1 & -.01 \\
11. A. Negativo & -.07 & $-.11^{*}$ & -.05 & $-.10^{*}$ & -.01 & $.13^{* *}$ & $.10^{*}$ & .01 & -.02 & .01 & 1 \\
\hline
\end{tabular}

Nota. Los coeficientes del grupo 1 se presentan por debajo de la diagonal; los coeficientes del grupo 2, por encima de la diagonal. El sexo fue codificado como 1 = varón y 2 = mujer; ${ }^{*} p<.05$; ${ }^{* *} p<.01$.

Tabla 4. Correlaciones bivariadas para el grupo 3 (15-17 años) y la muestra completa

\begin{tabular}{llllllllllll}
\hline & 1 & 2 & 3 & 4 & 5 & 6 & 7 & 8 & 9 & 10 & 11 \\
\hline 1. Sexo & 1 & $-.06^{*}$ & .00 & -.01 & -.02 & $-.17^{* *}$ & $-.11^{* *}$ & .00 & .04 & -.02 & .01 \\
2. Ap-Tarea & -.01 & 1 & $.57^{* *}$ & $.72^{* *}$ & $.53^{* *}$ & $.33^{* *}$ & $.37^{* *}$ & $.40^{* *}$ & $.41^{* *}$ & $.39^{* *}$ & $-.11^{* *}$ \\
3. Ev-Tarea & .01 & $.64^{* *}$ & 1 & $.53^{* *}$ & $.70^{* *}$ & $.18^{* *}$ & $.49^{* *}$ & $.25^{* *}$ & $.41^{* *}$ & $.30^{* *}$ & $-.08^{* *}$ \\
4. Ap-Yo & .01 & $.75^{* *}$ & $.57^{* *}$ & 1 & $.59^{* *}$ & $.32^{* *}$ & $.35^{* *}$ & $.39^{* *}$ & $.41^{* *}$ & $.36^{* *}$ & $-.08^{* *}$ \\
5. Ev-Yo & -.04 & $.56^{* *}$ & $.68^{* *}$ & $.59^{* *}$ & 1 & $.31^{* *}$ & $.58^{* *}$ & $.22^{* *}$ & $.37^{* *}$ & $.28^{* *}$ & -.03 \\
6. Ap-Otro & $-.13^{* *}$ & $.39^{* *}$ & $.25^{* *}$ & $.38^{* *}$ & $.38^{* *}$ & 1 & $.64^{* *}$ & $.20^{* *}$ & $.22^{* *}$ & $.20^{* *}$ & $.08^{* *}$ \\
7. Ev-Otro & $-.12^{* *}$ & $.41^{* *}$ & $.51^{* *}$ & $.37^{* *}$ & $.64^{* *}$ & $.67^{* *}$ & 1 & $.21^{* *}$ & $.34^{* *}$ & $.22^{* *}$ & $.05^{*}$ \\
8. Ap-Amistad & -.02 & $.40^{* *}$ & $.27^{* *}$ & $.39^{* *}$ & $.29^{* *}$ & $.24^{* *}$ & $.21^{* *}$ & 1 & $.63^{* *}$ & $.30^{* *}$ & .04 \\
9. Ev-Amistad & .03 & $.39^{* *}$ & $.42^{* *}$ & $.40^{* *}$ & $.40^{* *}$ & $.25^{* *}$ & $.32^{* *}$ & $.64^{* *}$ & 1 & $.29^{* *}$ & .03 \\
10. A. Positivo & .05 & $.37^{* *}$ & $.28^{* *}$ & $.35^{* *}$ & $.31^{* *}$ & $.19^{* *}$ & $.23^{* *}$ & $.31^{* *}$ & $.29^{* *}$ & 1 & -.02 \\
11. A. Negativo & .07 & $-.11^{* *}$ & $-.09^{*}$ & -.06 & -.03 & .00 & .00 & .00 & $.08^{*}$ & -.05 & 1
\end{tabular}

Nota. Los coeficientes para el grupo 3 se incluyen por debajo de la diagonal; los coeficientes para la muestra completa, por encima de la diagonal. El sexo fue codificado como $1=$ varón y $2=$ mujer. ${ }^{*} p<.05 ;{ }^{* *} p<.01$.

En segundo lugar, las seis metas de logro correlacionaron positiva y significativamente con las dos metas de amistad en todos los grupos de estudiantes. El grupo más joven obtuvo valores superiores entre las metas de evitación-tarea y evitación-amistad $(r=.45)$. En el grupo intermedio, las dos 
metas de amistad y la meta de aproximación-tarea obtuvieron valores más altos $(r=.38)$, al igual que la meta de evitación-amistad y aproximación-yo $(r=.38)$. Para finalizar, el grupo de mayor edad mostró valores similares al grupo más joven entre evitación-tarea y evitación-amistad $(r=.42)$.

En tercer lugar, todas las metas de logro correlacionaron positiva y significativamente con el afecto positivo. Sin embargo, el afecto negativo lo hizo de manera negativa con las metas orientadas a la tarea y al yo, y positivamente con las metas orientadas al otro. De manera unánime, el valor superior en los tres grupos de edad se da entre el afecto positivo y la aproximación-tarea, con valores que oscilan entre $r=.37$ y .40 , mientras que el inferior se encuentran entre el afecto negativo y aproximación-tarea $(r=-.11$ y -.10$)$.

Por último, en cuarto lugar, los valores de la muestra total son superiores a los reportados por las diferentes franjas de edad. Destaca la elevada correlación $(r=.72)$, entre las metas de aproximación-tarea y aproximación-yo, lo mismo ocurre con las metas de evitación-tarea y evitación-yo $(r=.70)$ y las metas de aproximación-otro y evitación-otro $(r=.64)$. En la relación entre aproximación-amistad y evitación-amistad se obtienen valores similares a los mostrados en las 3 franjas de edad $(r=63)$. En la afectividad, como en casos anteriores, las dos dimensiones adquieren valores prácticamente nulos.

\section{Diferencias relacionadas con la edad y el sexo}

Se realizó un MANOVA en el conjunto de las metas de logro y las variables aproximación-amistad, evitación-amistad, afecto positivo y afecto negativo, con los tres grupos de edad y el sexo como factores inter-sujetos. Se utilizó la Traza de Pillai en lugar de la Lambda de Wilks para determinar la significación multivariable, ya que no se cumplió el supuesto de homogeneidad de la matriz de covarianza, de acuerdo con la Prueba de Box (Tabachnick \& Fidell, 2001). Emergieron diferencias estadísticamente significativas en función del sexo $F$ (10, $1592)=7.39, p<.001\left(\eta^{2}=.04\right)$, del grupo de edad $F(20,3186)=4.65, p<.001$ $\left(\eta^{2}=.03\right)$, y de la interacción sexo y edad $F(20,3186)=1.63, p<.05\left(\eta^{2}=.010\right)$. 
Tabla 5. Análisis descriptivos de los datos en función del sexo para la muestra total y grupos de edad

\begin{tabular}{|c|c|c|c|c|c|c|c|c|c|}
\hline & \multirow[b]{2}{*}{ Sexo } & \multicolumn{2}{|c|}{$\begin{array}{l}\text { Muestra total } \\
\text { Varones: } 855 \\
\text { Mujeres: } 755\end{array}$} & \multicolumn{2}{|c|}{$\begin{array}{c}\text { Grupo } 1 \\
\text { (10-12 años) } \\
\text { Varones: } 195 \\
\text { Mujeres: } 210\end{array}$} & \multicolumn{2}{|c|}{$\begin{array}{c}\text { Grupo } 2 \\
\text { (13-14 años) } \\
\text { Varones: } 365 \\
\text { Mujeres: } 281\end{array}$} & \multicolumn{2}{|c|}{$\begin{array}{c}\text { Grupo } 3 \\
\text { (15-17 años) } \\
\text { Varones: } 295 \\
\text { Mujeres: } 264\end{array}$} \\
\hline & & $M$ & $D T$ & $M$ & $D T$ & $M$ & $D T$ & $M$ & $D T$ \\
\hline \multirow[t]{2}{*}{ Aproximación-tarea } & Varón & $4.17^{\star}$ & .74 & 4.33 & .68 & $4.22^{* *}$ & .73 & 4.02 & .76 \\
\hline & Mujer & 4.08 & .82 & 4.27 & .74 & 4.01 & .84 & 4.01 & .84 \\
\hline \multirow[t]{2}{*}{ Evitación-tarea } & Varón & 3.95 & .93 & 3.99 & .98 & 3.99 & .94 & 3.87 & .89 \\
\hline & $\mathrm{Mu}$ & 3.97 & .94 & 4.17 & .91 & 3.90 & .96 & 3.89 & .92 \\
\hline \multirow[t]{2}{*}{ Aproximación-yo } & Varón & 4.09 & .77 & 4.28 & .66 & 4.08 & .81 & 3.98 & .77 \\
\hline & & 4.07 & .83 & 4.28 & .73 & 3.98 & .85 & 4.00 & .84 \\
\hline \multirow[t]{2}{*}{ Evitación-yo } & Varón & 3.79 & .99 & 3.80 & 1.04 & $3.85^{\star}$ & .99 & 3.69 & .94 \\
\hline & Mujer & 3.73 & 1.02 & 3.94 & .97 & 3.69 & 1.01 & 3.60 & 1.05 \\
\hline \multirow[t]{2}{*}{ Aproximación-otro } & Varón & $3.40^{\star * \star}$ & 1.13 & $3.48^{\star * *}$ & 1.05 & $3.38^{* * *}$ & 1.15 & $3.37^{* *}$ & 1.16 \\
\hline & Mujer & 2.99 & 1.15 & 3.01 & 1.16 & 2.92 & 1.10 & 3.05 & 1.19 \\
\hline \multirow[t]{2}{*}{ Evitación-otro } & Varón & $3.56^{\star \star *}$ & 1.11 & $3.69^{*}$ & 1.09 & $3.57^{* *}$ & 1.11 & $3.46^{* *}$ & 1.11 \\
\hline & Mujer & 3.29 & 1.09 & 3.46 & 1.11 & 3.27 & 1.09 & 3.19 & 1.07 \\
\hline \multirow[t]{2}{*}{ Aproximación-amistad } & Varón & 3.91 & .91 & 4.19 & .77 & 3.84 & .98 & 3.82 & .90 \\
\hline & Mujer & 3.92 & .95 & 4.09 & .96 & 3.92 & .91 & 3.78 & .97 \\
\hline \multirow[t]{2}{*}{ Evitación-amistad } & Varón & 3.94 & .92 & 4.17 & .83 & 3.92 & .95 & 3.80 & .90 \\
\hline & Mujer & 4.02 & .93 & 4.27 & .89 & 3.98 & .92 & 3.85 & .93 \\
\hline \multirow[t]{2}{*}{ Afecto Positivo } & Varón & 2.41 & .35 & 2.48 & .32 & $2.41^{*}$ & .38 & 2.38 & .34 \\
\hline & Mujer & 2.40 & .35 & 2.44 & .34 & 2.34 & .37 & 2.42 & .34 \\
\hline \multirow[t]{2}{*}{ Afecto Negativo } & Varón & 1.75 & .44 & 1.78 & .44 & 1.76 & .45 & 1.72 & .42 \\
\hline & Mujer & 1.77 & .42 & 1.72 & .43 & 1.79 & .40 & 1.79 & .42 \\
\hline
\end{tabular}

Nota: ${ }^{*} p<.05,{ }^{* *} p<.01,{ }^{* * *} p<.001$

Los siguientes análisis univariados mostraron que los varones puntuaron más alto que las mujeres en las metas de aproximación-tarea, $F(1,1610)=5.74$, $p<.05\left(\eta^{2}=.004\right)$; aproximación-otro, $F(1,1610)=50.86, p<.001\left(\eta^{2}=.03\right)$, y evitación-otro, $F(1,1610)=22.59, p<.01\left(\eta^{2}=.01\right)$. La Tabla 5 muestra los estadísticos descriptivos de las variables a estudio en función del sexo para la muestra total y los tramos de edad. A diferencia de las otras horquillas de edad, de 13 a 14 años se dieron diferencias entre sexo en las metas de aproximacióntarea y evitación-yo en favor de los varones. En todas las franjas de edad se constataron las diferencias significativas en las metas de aproximación-otro y evitación-otro en favor de los varones.

Asimismo, se observaron diferencias en función de la edad en las metas de aproximación-tarea, $F(2,1609)=16.03, p<.001\left(\eta^{2}=.02\right)$; evitación-tarea, $F(2,1609)=5.48, p<.01\left(\eta^{2}=.01\right)$, aproximación-yo; $F(2,1609)=17.89, p<$ $.001\left(\eta^{2}=0.02\right)$; evitación-yo, $F(2,1609)=5.96, p<.01\left(\eta^{2}=.01\right)$, evitaciónotro, $F(2,1609)=5.97, p<.01\left(\eta^{2}=.01\right)$, aproximación-amistad, $F(2,1609)=$ 16.39, $p<.001\left(\eta^{2}=.02\right)$, evitación-amistad, $F(2,1609)=22.47, p<.001\left(\eta^{2}=\right.$ $0.03)$, y afecto positivo, $F(2,1609)=6.53, p<.01\left(\eta^{2}=.01\right)$. El análisis Post Hoc de Scheffé mostró que, en general, el peso de las metas de logro decrece con el aumento de la edad de los estudiantes (Tabla 2). Todas las comparaciones pareadas se mostraron estadísticamente significativas $(p<.05)$. Se encontraron tres patrones en todos los tramos de edad: (a) en relación a las metas de logro: aproximación-tarea > aproximación-yo > evitación-tarea > evitación-yo > evitación-otro > aproximación-otro; (b) en cuanto a las metas de amistad: 
evitación-amistad > aproximación amistad, y (c) en la afectividad: afecto positivo $>$ afecto negativo.

Por último, se observó una interacción significativa entre sexo y edad en afecto positivo, $F(2,1609)=3.24, p<.05\left(\eta^{2}=.004\right)$. Si bien, tanto en varones como en mujeres, se observa una caída de los valores en esta variable con el incremento de la edad, también tiende a ser menor en las diferencias de sexo.

\section{Análisis de regresión lineal}

En la Tabla 6 se muestran los análisis de regresión lineal realizados para examinar las metas de logro $3 \times 2$ y las metas de amistad como predictores de las variables dependientes (afectividad positiva y negativa). Se llevaron a cabo ocho análisis de regresión, dos para la muestra total y dos por cada tramo de edad. Dada la gran cantidad de análisis de regresión, por razones de claridad expositiva, únicamente se exponen los valores de $\beta$. Los análisis preliminares mostraron que el factor de inflación de la varianza (FIV) para las metas de logro variaba entre 1.98 y 2.62 (muy por debajo del criterio de corte convencional de $10)$, y que los valores de Tolerancia oscilaban entre .37 y .50 , lo indica que se cumple el supuesto de no multicolinealidad. El valor de la prueba de DurbinWatson (entre 1.70 y 1.89 para el afecto positivo, y 1.85 y 2.12 para el afecto negativo en los tramos de edad), también permitió aceptar el supuesto de independencia de errores, siendo aceptables valores entre 1 y 3.

El análisis en la muestra total reveló que las metas de aproximación-tarea y aproximación-amistad eran los principales predictores positivos y significativos del afecto positivo, junto con una predicción de menor peso de las metas de aproximación-yo y evitación-tarea $\left(R^{2}=.20\right)$. Por grupos de edad se constataron variaciones; el análisis mostró cómo de los 13 a 14 años las tres metas de aproximación de logro y las de evitación-tarea eran los principales predictores positivos y significativos del afecto positivo $\left(R^{2}=.21\right)$; mientras que en el grupo 1 y 3 lo fueron las metas de aproximación-tarea y aproximación-amistad $\left(R^{2}=\right.$ .21 y .19 , respectivamente).

Tabla 6. Metas de logro y metas de amistad como predictores de la afectividad en cada grupo de edad

\begin{tabular}{|c|c|c|c|c|c|c|c|c|}
\hline & \multicolumn{2}{|c|}{$\begin{array}{l}\text { Muestra total } \\
(n=1610)\end{array}$} & \multicolumn{2}{|c|}{$\begin{array}{c}10-12 \text { años } \\
(\mathrm{n}=405)\end{array}$} & \multicolumn{2}{|c|}{$\begin{array}{c}13-14 \text { años } \\
(\mathrm{n}=646)\end{array}$} & \multicolumn{2}{|c|}{$\begin{array}{c}15-17 \text { años } \\
(\mathrm{n}=559)\end{array}$} \\
\hline & $\begin{array}{c}\text { Afecto } \\
\text { Positivo }\end{array}$ & $\begin{array}{c}\text { Afecto } \\
\text { Negativo }\end{array}$ & $\begin{array}{c}\text { Afecto } \\
\text { Positivo }\end{array}$ & $\begin{array}{c}\text { Afecto } \\
\text { Negativo }\end{array}$ & $\begin{array}{l}\text { Afecto } \\
\text { Positivo }\end{array}$ & $\begin{array}{c}\text { Afecto } \\
\text { Negativo }\end{array}$ & $\begin{array}{c}\text { Afecto } \\
\text { Positivo }\end{array}$ & $\begin{array}{c}\text { Afecto } \\
\text { Negativo }\end{array}$ \\
\hline & $\beta$ & $\beta$ & $\beta$ & $\beta$ & $\beta$ & $\beta$ & $\beta$ & $\beta$ \\
\hline Ap-tarea & $.19^{\star \star \star}$ & $-.14^{* \star \star}$ & $.20^{* \star}$ & $-.14^{*}$ & $.19^{\star \star \star}$ & $-.13^{\star}$ & $.18^{* \star}$ & $-.15^{*}$ \\
\hline Ev-tarea & $.08^{*}$ & $-.09^{*}$ & .12 & -.04 & $.16^{\star \star}$ & -.12 & -.02 & -.12 \\
\hline Ap-yo & $.09^{*}$ & -.06 & .03 & -.08 & $.14^{*}$ & -.12 & .08 & .04 \\
\hline Ev-yo & .02 & .05 & .01 & .03 & -.07 & .12 & .10 & .02 \\
\hline Ap-otro & .06 & $.10^{* *}$ & .02 & .13 & $.14^{* *}$ & $.15^{\star *}$ & -.01 & .01 \\
\hline Ev-otro & -.01 & .04 & .07 & .10 & -.10 & .02 & .03 & .04 \\
\hline Ap-amistad & $.12^{\star * \star}$ & $.07^{*}$ & $.18^{\star *}$ & .06 & .06 & $.18^{\star *}$ & $.14^{\star *}$ & -.06 \\
\hline Ev-amistad & .05 & .06 & .01 & -.00 & .07 & -.02 & .06 & $.19^{\star *}$ \\
\hline$R^{2}$ & $.20^{\star \star \star}$ & $.04^{\star \star *}$ & $.21^{\star \star \star}$ & $.05^{\star \star}$ & $.21^{\star \star *}$ & $.07^{\star \star}$ & $.19^{\star \star \star}$ & $.04^{\star *}$ \\
\hline
\end{tabular}


En cuanto segundo lugar, y considerando la muestra total, los análisis revelaron que las metas de aproximación-tarea (en negativo) y las metas de aproximación-otro y aproximación-amistad (ambas en positivo) eran predictores significativos del afecto negativo $\left(R^{2}=.04\right)$. Este patrón se refleja en la franja de 13 a 14 años $\left(R^{2}=.07\right)$, mientras que en los grupos 1 y 3 sólo se mantuvieron como predictor negativo las metas de aproximación-tarea si bien de los 15-17 años también emergieron las metas de evitación-amistad como predictores positivos del afecto negativo ( $R^{2}=.05$ y .04 , respectivamente).

\section{DISCUSIÓN}

El presente estudio se propone tres objetivos: (a) analizar las diferencias relativas a la edad y el sexo en las metas de logro $3 \times 2$, las metas de amistad (aproximación y evitación), y la afectividad en el contexto de la Educación Física, (b) estudiar las relaciones entre las propias metas de logro 3x2 y con las otras variables a estudio, y (c) explorar el valor predictivo conjunto de metas de logro $3 \times 2$ y las metas de amistad en la afectividad en función de la edad.

Respecto al análisis de las diferencias relativas al desarrollo, se encontró una propensión de los estudiantes de menor edad a puntuar más alto las variables a estudio, a excepción de las metas de aproximación-otro y el afecto negativo, en las que no hubo diferencias (e.g., Bong, 2009; Shim, Ryan, \& Anderson, 2008). Igualmente, se observó una tendencia a respuestas menos fiables de los estudiantes más jóvenes que de los estudiantes de mayor edad. Ambos patrones asociados al desarrollo han sido descritos en estudios previos (e.g., Bong, 2009). En segundo lugar, se encontraron patrones idénticos en los tres tramos de edad en relación a la fuerza de la adopción de las metas de logro. Las metas de aproximación-tarea junto con las metas de aproximación-yo fueron las más valoradas, seguidas de las metas de evitación (tarea, yo, y otro, respectivamente), y por último, las metas de aproximación-otro. Estos resultados son congruentes con los reportados en el contexto académico general desde la perspectiva de metas de logro 3x2 (Méndez-Giménez et al., 2017). Los resultados revelaron la adopción de metas múltiples en estos grupos de estudiantes de Educación Física, con prioridad de las metas de aproximacióntarea y yo, y menor énfasis en las metas relativas a la comparación con los demás. Estos resultados también son consistentes con la investigación realizada desde los paradigmas de metas de logro preliminares (modelo dicotómico al 2x2) en el contexto de la Educación Física (e.g., Cecchini et al., 2008; Cecchini et al., 2011; Moreno-Murcia, Cervelló, \& González-Cutre, 2008). Sin embargo, divergen de los encontrados en el estudio de Bong (2009), donde los estudiantes coreanos a partir de $5^{\circ}$ y $6^{\circ}$ de E. Primaria y superiores, adoptaron con más fuerza las metas de aproximación-rendimiento en el contexto de las clases matemáticas. Como señalaron Dekker et al. (2013), el contexto sociocultural puede ejercer una influencia en la fuerza de la adopción de las metas por parte de los estudiantes.

Todos los tipos de metas de logro disminuyeron a través del tiempo excepto las metas de aproximación-otro, que permanecieron invariables en las tres etapas de la adolescencia. La investigación previa ha mostrado cómo las metas de maestría descienden con la edad, al menos hasta los 15 años (Bong, 
2009; Dekker et al, 2013; Digelidis \& Papaioannou, 1999; Wang \& Pomerantz, 2009; Wigfield \& Cambria, 2010). Ruble, Boggiano, Feldman y Loebl (1980) mostraron que los niños de menor edad se centran más en la tarea que están realizando que en el rendimiento de los demás, por ello, asignan menos valor a la comparación social, es decir, a las metas relacionadas con los otros. Generalmente, el ambiente escolar en cursos superiores se centra más en la adquisición de conocimientos y en aprendizajes orientados a una mayor comparativa con los demás. Los estudiantes perciben en el ambiente un mayor énfasis en la comparación social, la evaluación y el rendimiento según progresan en los cursos de educación secundaria lo que podría influir en su adopción de metas de logro (e.g., Urdan \& Midgley, 2003). Los resultados del presente estudio podrían apuntar que esa influencia contextual es la que provoca que no decaigan las puntaciones las metas de aproximación-otro en la misma manera en que lo hace el resto de metas. Futuros trabajos deberían estudiar la posible interacción entre el desarrollo y el ambiente en la persecución de las metas de logro de los adolescentes.

Por otro lado, las metas de evitación-amistad fueron superiores a las de aproximación-amistad. Diversos estudios han corroborado este patrón en la adolescencia en que el miedo a una incompetencia social es más fuerte que la lucha por conseguir esa competencia (e.g., Garn \& Sun, 2009; MéndezGiménez, Cecchini \& Fernández-Río, 2012; Méndez-Giménez, Fernández-Río \& Méndez-Alonso, 2015). Asimismo el afecto positivo superó al afecto negativo en todas las franjas de edad, lo que pone de manifiesto el balance positivo en términos afectivo-emocionales que ejerce la asignatura de EF entre los estudiantes.

Respecto a la comparativa en función del sexo, se encontraron diferencias significativas en tres de las seis metas de logro. Concretamente, los varones puntuaron más alto las metas de aproximación-tarea, aproximación-otro y evitación-otro que las mujeres. Centrándose en las franjas de edad, las puntuaciones más altas de las metas de aproximación-tarea en los varones de la muestra se explican por un descenso más parsimonioso en la fuerza de la adopción de estas metas en los varones que en las mujeres. De esta manera, a los 10-12 años no se encontraban diferencias entre sexos; en la franja de 13-14 años, la fuerza con que las mujeres adoptaron estas metas descendió lo que provocó las diferencias en favor de los varones, y finalmente las diferencias volvieron a desaparecer a partir de los 15 años, tras el descenso en los varones. Algo similar ocurrió en las metas de evitación-yo. Por otro lado, existe una gran evidencia que refuerza la idea de que los varones están más orientados a la comparación y superación de los propios compañeros que las chicas, tanto en el contexto académico general como de la Educación Física (e.g. Dekker et al., 2013; Moreno-Murcia et al., 2008; Schwinger \& Wild, 2012; Wu, 2012).

En relación al segundo objetivo, concretamente respecto a las relaciones entre las seis metas de logro se puede observar cómo los valores de los estudiantes más jóvenes tienden a ser inferiores a los de mayor edad, estos datos son coincidentes con los reportados por Bong (2009) con encuestados similares a los de este estudio. Las correlaciones más elevadas entre algunas 
metas, como es el caso de aproximación-tarea y aproximación-yo, son asumibles teniendo en cuenta la evolución del modelo de metas de logro 3x2. Pese a ello, Elliot et al. (2011) defendieron la necesidad de diferenciar ambos constructos y mostraron diferentes relaciones con variables de resultados. En cuanto a las relaciones de las metas de logro con otras variables, se observa cómo todas las metas de logro correlacionaron positivamente con las metas de amistad en las tres franjas de edad. Estos datos son congruentes con los reportados por Méndez-Giménez et al. (2012) y Garn \& Sun (2009) desde el modelo de metas $2 \times 2$ y refuerzan la importancia de combinar las dimensiones de logro y social para comprender el comportamiento motivacional de los niños y adolescentes en el ámbito de la actividad física y el deporte.

En relación al tercer objetivo, en los grupos de menor y mayor edad, las metas de aproximación-tarea y aproximación-amistad fueron los únicos predictores del afecto positivo. Sin embargo, en el grupo de 13 a 14 años, además de las metas de aproximación-tarea, emergieron otros predictores como las metas de aproximación-yo, aproximación-otro, y evitación-tarea. Por otro lado, en los grupos de menor y mayor edad, las metas de aproximación-tarea (en negativo) fueron los únicos predictores del afecto negativo, junto con las metas de evitación-amistad. Sin embargo, en el grupo de 13-14 años, las metas de aproximación-tarea (en negativo), y las metas de aproximación-otro, y aproximación-amistad (en positivo) fueron los predictores del afecto negativo. Estos resultados ponen de relieve la inestabilidad emocional y socioafectiva por la que pasan los adolescentes, especialmente en la etapa de los 13-14 años, y el enorme peso que tienen las relaciones entre iguales en la motivación de los estudiantes y sus consecuencias motivacionales. En este periodo en que las relaciones sociales son tan relevantes, la búsqueda de amistades y los esfuerzos de competencia basada en la comparación con el otro pueden provocar afectos ambivalentes y reacciones confusas.

Una limitación del estudio es su carácter transversal. Futuras investigaciones podrían considerar diseños longitudinales.

\section{REFERENCIAS BIBLIOGRÁFICAS}

Ames, C. (1984). Competitive, cooperative, and individualistic goal structures: A cognitive-motivational analysis. En C. Ames \& R. Ames (Eds.), Research on motivation in education (Vol. 3, pp. 177-207). New York: Academic Press.

Ames, C. (1992). Classrooms: Goals, structure, and student motivation. Journal of Educational Psychology, 84, 261-271. http://dx.doi.org/10.1037/0022$\underline{0663.84 .3 .261}$

Bong, M. (2009). Age-related differences in achievement goal differentiation. Journal of Educational Psychology, 101, 879-896. http://dx.doi.org/10.1037/a0015945

Brondino, M., Raccanello, D., \& Pasini, M. (2014). Achievement goals as antecedents of achievement emotions: The 3x2 achievement goal model as a framework for learning environments design. En T. D. Mascio, R. Gennari, P. Vittorini, R. Vicari, y F. de la Prieta (Eds.), Methodologies and intelligent 
systems for technology enhanced earning (pp. 53-60). Switzerland: Springer International Publishing.

Cecchini, J. A., González, C., Méndez-Giménez, A. Fernández-Río, J., Contreras, O., \& Romero, S. (2008). Metas sociales y de logro, persistenciaesfuerzo e intenciones de práctica deportiva en el alumnado de Educación Física. Psicothema, 20(2), 260-265.

Cecchini, J. A. González, C., Méndez-Giménez, A., \& Fernández-Río, J. (2011). Achievement goals, social goals, and motivational regulations reported by students in physical education settings. Psicothema, 23(1), 51-57.

Dekker, S., Krabbendam, L., Lee, N. C., Boschloo, A., de Groot, R., \& Jolles, J. (2013). Sex differences in goal orientation in adolescents aged 10-19: The older boys adopt work-avoidant goals twice as often as girls. Learning and Individual Differences, 26 ,

196-200. http://dx.doi.org/10.1016/i.lindif.2012.07.011

Digelidis, N., \& Papaioannou, A. (1999). Age-group differences in intrinsic motivation, goal orientations and perceptions of athletic competence, physical appearance and motivational climate in Greek physical education. Scandinavian Journal of Medicine \& Science in Sports, 9, 375-380. http://dx.doi.org/10.1111/j.1600-0838.1999.tb00259.x

Diseth, A. (2015). The advantages of task-based and other-based achievement goals as standards of competence. International Journal of Educational Research, 72, 59-69. http://dx.doi.org/10.1016/j.ijer.2015.04.011

Dweck, C. S. (1986). Motivational processes affecting learning. American Psychologist, 41, 1040-1048. http://dx.doi.org/10.1037/0003066X.41.10.1040

Dweck, C. S. (2000). Self-theories: Their role in motivation, personality, and development. Philadelphia, PA: Psychology Press.

Elliot, A. J., \& Church, M. A. (1997). A hierarchical model of approach and avoidance achievement motivation. Journal of Personality and Social Psychology, 72, 218-232. http://dx.doi.org/10.1037/0022-3514.72.1.218

Elliot, A. J., \& Harackiewicz, J. M. (1996). Approach and avoidance achievement goals and intrinsic motivation: A mediational analysis. Journal of Personality and Social Psychology, 70, 461-475. http://dx.doi.org/10.1037/00223514.70.3.461

Elliot, A. J., \& McGregor, H. A. (2001). A 2 × 2 achievement goal framework. Journal of Personality and Social Psychology, 80(3), 501-519. http://dx.doi.org/10.1037/0022-3514.80.3.501

Elliot, A. J., Murayama, K., \& Pekrun, R. (2011). A $3 \times 2$ achievement goal model. Journal of Educational Psychology, 103(3), 632-648. http://dx.doi.org/10.1037/a0023952

Garn, A. C., \& Sun, H. (2009). Approach-Avoidance motivational profiles in early adolescents to the PACER fitness test. Journal of Teaching in Physical Education, 28, 400-421. http://dx.doi.org/10.1123/itpe.28.4.400

Juvonen, J., Le, V., Kaganoff, T., Augustine, C., \& Constant, L. (2004). Focus on the wonder years: Challenges facing the American middle school. Santa Monica, CA: RAND Corporation.

Maehr, M. L. (1989). Thoughts about motivation. En C. Ames y R. Ames (Eds.), Research on motivation in education (Vol. 3, pp. 299-315). New York: Academic Press. 
Mascret, N., Elliot, A. J., y Cury, F. (2015). Extending the $3 \times 2$ achievement goal model to the sport domain: The $3 \times 2$ achievement goal questionnaire for sport. Psychology of Sport and Exercise, 17(1), 7-14. http://dx.doi.org/10.1016/j.psychsport.2014.11.001

Méndez-Giménez, A., Cecchini, J. A., \& Fernández-Río J. (2014). Examinando el modelo de metas de logro $3 \times 2$ en el contexto de la Educación Física. Cuadernos de Psicología del Deporte, 14(3), 157-167.

Méndez-Giménez, A., Cecchini, J.A., Fernández-Río, J., Méndez-Alonso, D., \& Prieto-Saborit, J.A. (2017). Metas de logro 3x2, motivación autodeterminada y satisfacción con la vida en Educación Secundaria. Revista de Psicodidáctica, $22(2)$, 150-156. http://dx.doi.org/10.1016/i.psicod.2017.05.001

Méndez-Giménez, A., Fernández-Río, J., \& Cecchini, J. A. (2012). Análisis de un modelo multiteórico de metas de logro, metas de amistad y autodeterminación en educación física. Estudios de Psicología, 33(3), 325336.

Méndez-Giménez, A., Fernández-Río. J., \& Cecchini, J.A. (2014). Validación de la versión en español del Cuestionario de Metas de Amistad en Educación Física. Universitas Psychologica, 13(1), 227-237. http://dx.doi.org/10.11144/Javeriana.UPSY13-1.vvec

Méndez-Giménez, A., Fernández-Río, J., \& Cecchini, J.A. (2015). Perfiles motivacionales de aproximación-evitación en contextos de educación física. Universitas Psychologica, 14(2), 549-562. http://dx.doi.org.10.11144/Javeriana.upsy14-2.pmae

Méndez-Giménez, A., Fernández-Río, J., \& Méndez-Alonso (2015). Modelo de Educación Deportiva versus Modelo Tradicional: Efectos en la motivación y deportividad. Revista Internacional de Medicina y Ciencias de la Actividad $\begin{array}{llll}\text { Física } y & \text { el } & \text { Deporte, } & \text { 15(59), }\end{array}$ http://dx.doi.org/10.15366/rimcafd2015.59.004

Moreno-Murcia, J.A., Cervelló, E., \& González-Cutre, D. (2008). Relationships among Goal Orientations, Motivational Climate and Flow in adolescent athletes: differences by gender. The Spanish Journal of Psychology, 11(1), 181-191.

Nicholls, J. G. (1984). Achievement motivation: Conceptions of ability, subjective experiences, task choice and performance. Psychological Review, 91, 328346. http://dx.doi.org/10.1037/0033-295X.91.3.328

Nunnally, J. C. (1978). Psychometric theory. New York: McGraw-Hill.

Paulick, I., Watermann, R., \&, Nückles, M. (2013). Achievement goals and school achievement: The transition to different school tracks in secondary school. Contemporary Educational Psychology 38, 75-86. http://dx.doi.org/10.1016/j.cedpsych.2012.10.003

Ruble, D. N., Boggiano, A. K., Feldman, N. S., \& Loebl, J. H. (1980). Developmental analysis of the role of social comparison in selfevaluation. Developmental Psychology, 16, 105-115. http://dx.doi.org/10.1037/00121649.16.2.105

Sandín, B. (2003). Escalas PANAS de afecto positivo y negativo para niños y adolescentes (PANASN). Revista de Psicopatología y Psicología Clínica, 8(2), 173-182.

Schwinger, M., \& Wild, E. (2012). Prevalence, stability, and functionality of achievement goal profiles in mathematics from third to seventh grade. 
Contemporary Educational Psychology, 37(1), 1-13. http://dx.doi.org/10.1016/j.cedpsych.2011.08.001

Shim, S. S., Ryan, A. M., \& Anderson, C. J. (2008). Achievement goals and achievement during early adolescence. Examining time-varying predictors and outcome variables in growth-curve analysis. Journal of Educational Psychology, 100(3), 655-671. h http://dx.doi.org/10.1037/00220663.100.3.655

Tabachnick, B. G., \& Fidell, L. S. (2001). Using multivariate statistics (4th Ed.). Boston: Allyn and Bacon.

Urdan, T., \& Midgley, C. (2003). Changes in the perceived classroom goal structure and pattern of adaptive learning during early adolescence. Contemporary Educational Psychology, 28, 524-551. http://dx.doi.org/10.1016/S0361-476X(02)00060-7

Wang, Q., \& Pomerantz, E. M. (2009). The motivational landscape of early adolescence in the United States and China: A longitudinal investigation. Child Development, 80(4), 1272-1287. http://dx.doi.org/10.1111/j.14678624.2009.01331.X

Wigfield, A., \& Cambria, J. (2010). Students' achievement values, goal orientations, and interest: Definitions, development, and relations to achievement outcomes. Developmental Review, 30(1), 1-35. http://dx.doi.org/10.1016/j.dr.2009.12.001

Wu, C. C. (2012). The cross-cultural examination of $3 \times 2$ achievement goal model in Taiwan. Procedia-Social and Behavioral Science, 69, 422-427. http://dx.doi.org/10.1016/j.sbspro.2012.11.429

Número de citas totales / Total references: 38 (100\%)

Número de citas propias de la revista / Journal's own references: 1 (2,63\%)

Rev.int.med.cienc.act.fís.deporte - vol. 18 - número 72 - ISSN: 1577-0354 